polysulphide, the former determined as stannic oxide and the latter electrolytically. Arsenic was separated from copper by Crookes' method, and sulphur was weighed as barium sulphate after oxidation with nitric acid in a sealed tube.

I. Museum No. I842. Small chisel or pinch-bar, $18 \times \mathrm{I}^{1 / 8} \times 1 / 2$ inches. Very tough. Density, 8.68.

II. Museum No. B-I840. Implement $5^{-6}$ inches long, very hard and tough; pale color. Density, 8.94.

III. Museum No. 1959. Thick wide chisel $4 \frac{1}{2}$ inches long, tough but less hard. Density, 8.92.

IV. Museum No. I-859. Socketed spear-head, I2 inches long. Density, 8.89 .

V. Museum No. 24I3. F agment of pointed bar 6 inches long. Density, 8.6r.

VI. Museum No. I949. Small cast chisel; contained characteristic air-holes or "pipes." Apparently contained considerable oxide. Density, 8. I8(?).

\begin{tabular}{|c|c|c|c|c|c|c|}
\hline & I. ${ }^{1}$ & II. & $\begin{array}{l}\text { ANALYSIS. } \\
\text { III. }{ }^{1}\end{array}$ & IV. & $\mathrm{V} .{ }^{2}$ & VI. \\
\hline $\mathrm{Cu} . . . .$. & $9 \mathrm{I} .8 \mathrm{I}$ & $90.5 \mathrm{I}$ & $95 \cdot 59$ & $97 \cdot 43$ & 94.96 & $9 \mathrm{I} \cdot 43$ \\
\hline Sn. . . . . & $7 \cdot 56$ & 8.92 & 4.48 & $\ldots \ldots$ & 4.98 & 7.05 \\
\hline $\mathrm{Pb} \ldots \ldots$ & $\ldots \ldots$ & $\ldots \ldots$ & $\ldots \ldots$ & trace (?) & $\ldots \ldots$ & $\ldots$ \\
\hline $\mathrm{Fe} \ldots \ldots$ & trace & trace & trace & trace & $\ldots \ldots$ & trace \\
\hline S....... & $\ldots$ & trace & $\ldots$ & little & 0.53 & $\ldots$ \\
\hline As...... & $\ldots \ldots$ & $\ldots \ldots$ & $\ldots \ldots$ & 2.14 & $\ldots \ldots$ & $\ldots \ldots$ \\
\hline & $99 \cdot 37$ & 99.43 & 100.07 & 99.57 & 100.47 & $98 \cdot 4^{8}$ \\
\hline
\end{tabular}

To this report may be added the record of an analysis, made in I9or, by Dr. A. E. Hill with one of us, of a figurine found in Honduras. Color, pale yellow; density, 8.94-6. Cu 93.19, Sn I.64, $\mathrm{Pb}$ I.60, Fe 0.40 per cent.; $\mathrm{Au}, \mathrm{Sb}$ and $\mathrm{Zn}$ absent.

New YoRk, February, I9Io.

[Contributions from the LABORATORy of Physical Chemistry, University of ILIINOIS.]

\title{
THE FUNDAMENTAL LAW FOR A GENERAL THEORY OF SOLUTIONS. ${ }^{1}$ \\ BY EDWARD W. WASHBURN. \\ Received March 3 , 1910. \\ Nomenclature.
}

C

Volume concentration.

(1) $C_{P}$, (2) $c_{p} \quad$ Molecular heat capacity of (1) a liquid, (2) a gas.

1 Average of two complete analyses.

2 Average of three concordant analyses.

1 Presented at the Second Decennial Celebration of Clark University, Worcester, Mass., September 16, I909. 


\section{$\Delta C_{P}$}

(1) $K_{C},(2) K_{N}$

$K_{S}$

(1) $L_{S}(2) L_{v}$ (3) $L_{F}$

(1) $N,(2) N^{\prime}$

(I) $n,(2) n^{\prime}$

$p$

$P$

$Q_{\pi}$

$R$

(I) $T$, (2) $T_{F}$, (3) $T_{0}$,

(4) $T_{B}$, (5) $T_{B 0}$

$U$

(I) $v,(2) \mathrm{V}$

$\pi$

$\mu$

$\psi$

$\xi$

Decrease in molecular heat capacity attending a change in state of aggregation.

Equilibrium constant in terms of (I) volume concentrations, (2) mol fractions. (Products of the reaction in the denominator.)

Solubility product in terms of mol fractions.

Molecular heat of (I) sublimation, (2) vaporization, (3) fusion (under constant external pressure).

Mol fraction of (1) solvent, (2) solute.

Number of mols of (I) solvent, (2) solute.

Gas or vapor pressure.

External pressure on a liquid or solid.

Heat evolved when the reaction $a \mathrm{~A}+b \mathrm{~B}+\ldots=m \mathrm{M}+$ $n \mathrm{~N}+\ldots$ takes place from left to right in a solution under osmotic equilibrium.

Gas constant.

(1) Absolute temperature, (2) absolute temperature of the freezing point of a solution, (3) of the freezing point of the pure solvent, (4) of the boiling point of a solution, (5) of the boiling point of the pure solvent.

Total energy decrease produced when the reaction $a \mathrm{~A}+b \mathrm{~B}+$ $=m \mathrm{M}+n \mathrm{~N}+\ldots$ takes place from left to right.

Molecular volume of (I) a gas, (2) a liquid.

Osmotic pressure.

Thermodynamic potential.

Fugacity.

Activity.

I. The Development of the Modern Theory of Solutions.

Upon the foundations laid by the labors of van't Hoff and Arrhenius has arisen the structure which we know to-day as the Modern Theory of Solutions. Before van't Hoff's epoch-making discovery of the thermodynamic relations which bind together the colligative properties ${ }^{2}$ of dilute solutions, our knowledge concerning these important quantities was confined to a set of apparently unconnected empirical laws. Van't Hoff's generalization of these laws, followed almost immediately by the Ionic Theory of Arrhenius, stimulated greatly the study of solutions and made possible the rapid development and perfection of our present theory. Investigation has, however, been confined chiefly to the domain of dilute solutions and the Modern Theory of Solutions has remained almost entirely a theory of dilute solutions. The reason for this is, I believe, due largely to one of those historical "accidents" which occur now and then in the development of science. The history of this "accident" and the manner in which it came about forms a chapter in physical chemistry of interest alike to the chemist and to the philosopher.

Perhaps the best way to form a clear idea of the process of evolution of our present theory of solutions is to consider first the colligative properties of solutions and the relations which connect them. These quantities-the osmotic pressure, vapor pressure, freezing point, boiling point,

2Following Ostwald, the "a "colligative properties" is used to embrace such properties as osmotic pressure, boiling point raising, freezing point lowering, vapor pressure lowering, etc. 
etc. - have played such an important and vital part in the development of our solution theory that a clear idea of their relations to one another is absolutely essential to a proper understanding of the theory and of its development. The nature of these relations is expressed by the following statement: The colligative properties of a solution are connected by a set of rigorous differential equations which involve no assumptions except the two laws of thermodynamics. The equations are as follows:

(I) Osmotic Pressure and Freezing Point,

$$
d \pi=\left(\frac{-L_{F}}{V}\right) \frac{d T_{F}}{T_{F}} .
$$

(2) Vapor Pressure and Freezing Point,

$$
d p=\left(\frac{L_{S}}{v}\right) \frac{d T_{F}}{T_{F}}
$$

(3) Osmotic Pressure and Vapor Pressure,

$$
d \pi=\left(\begin{array}{c}
-v \\
V
\end{array}\right) d p .
$$

(4) Osmotic Pressure and Boiling Point,

$$
d \pi=\left(\frac{L_{v}}{V}\right) \frac{d T_{B}}{T_{B}} .
$$

To these should be added a number of others, such as the relation between the electromotive force of a concentration cell and either vapor pressure (74) or osmotic pressure (70), and ( 16 and 14 ) the mutual relations among the osmotic pressures or vapor pressures of the constituents of a physical mixture or ( 30 and $30 a$ ) the substances concerned in a chemical equilibrium, etc. ${ }^{3}$ While the following discussion applies with equal force to all of these relations, it will perhaps be conducive to clearness, if we confine our attention chiefly to the four relations given above.

From the method of derivation of these relations, it is clear that they do not involve any assumptions regarding the concentration of the solution, nor do they depend in any way upon the nature of the dissolved solute or its degree of association, dissociation, or solvation. In fact, if one knows, for example, the vapor pressure, freezing point lowering or boiling point raising for a solution of any nonvolatile solute, he has the means of calculating the osmotic pressure for the same temperature without knowing either the concentration of the solution or the nature of the solute. There may be one or several solutes present and they may associate, dissociate, or unite with each other or with the solvent in any manner and to any extent. These questions are in no way involved in the calculation. Since relations such as those existing among the colligative properties of a solution involve only the two laws of thermodynamics, it will be convenient to refer to them as "purely thermodynamic relations," to distingtiish them from an important group of relations which

${ }^{3}$ The derivations of these relations and a more detailed discussion of them are given in a previous paper, "A Simple System of Thermodynamic Chemistry Based upon a Modification of the Method of Carnot" (This Journal, 32, 467 (I9IO)). For convenience in reference these equations are given the same number here as in the previous paper where the significance of the quantities appearing in the equations is explained in detail. 
involve the composition of the solution and the nature of its components, and which we will now proceed to consider.

Let us consider a solution of any solute $A$ in any solvent $B$, and let our problem be to express each of the colligative properties of the solution as a function of its composition or its "concentration." This problem can in general be solved only by direct experiment for the particular solvent and solute under consideration. From what has preceded, however, it is evident that as soon as we know the relation between any one of the colligative properties and the composition of the solution, the other relations become thereby determined. If, for example, we determined the freezing point of the solution for a series of concentrations, we could calculate thermodynamically the osmotic pressure, the vapor pressure, etc., for the same concentrations and thus derive an equation connecting each of these quantities with the concentration. The colligative property which should be chosen for experimental study in a given case would depend upon the relative ease and accuracy with which the several quantities could be determined and the temperature range which it was desired to cover; also in some cases upon whether the requisite "caloric quantities"' were known with sufficient accuracy or could be determined readily.

The relation between any one of the colligative properties and the concentration of the solution for any given solvent and solute will obviously depend upon the degree of association, dissociation and solvation of the solute. ${ }^{5}$ Since the magnitude of these effects and their dependence upon the concentration are in general unknown quantities, the problem is too complex for any complete solution. In general, therefore, it is necessary to make a separate investigation for every solvent and solute in order to establish the desired relation connecting some one of the colligative properties with the concentration. To leave the problem in this condition, however, is naturally not very satisfactory, and the course usually followed by science when confronted with a problem which is too complex is first to simplify the problem. Let us try to trace the process of simplification which has been followed by science in the present instance.

Since association or dissociation of either solvent or solute molecules introduces complications, the first step in the process of simplification is obviously to consider the simple case of a solution in which neither takes place. Since the union of a portion or all of the solute molecules with the solvent molecules (solvation) is also a complicating factor, the next step in the process of simplification would naturally be to eliminate this factor also by assuming no solvation. After making these simplifications our problem would read as follows: What are the relations connecting the colligative properties with the composition in the case of a solution in which the number of molecular species present is equal to the number of components?" Let us call such a solution, provisionally, an "ideal solution," postponing until later a more definite and accurate description

${ }^{4}$ Proposed by van der Waals to distinguish heat capacities, latent heats, heats of reaction, etc., from colligative properties.

${ }^{5}$ It is hardly necessary to remark that this statement tacitly assumes that the desired relation is to be one which involves the number of mols of the solute.

' For example, in the case of two components, a solvent and one solute, there would be only two different kinds of molecules; for a solvent and two solutes, only three different kinds of molecules, etc. 
of the properties of the type of solution to which the term "ideal" should be applied.

There is, however, another method by which the complicating factor of solvation can also be eliminated. Willard Gibbs, in his monumental work on thermodynamic chemistry, has taught us that the proper way to represent the composition of any phase is by the means of the mol fractions of its several components. ${ }^{\text {? }}$

Now in the case of a solution, the mol fraction of the solute (for example) will be altered if it becomes solvated on going into solution, owing to the consequent change in the number of solvent molecules. Such a complication can be eliminated from our problem, as explained above, by assuming no solvation; or it can be likewise eliminated by taking the solution sufficiently dilute. For, as the solution becomes more and more dilute, the limit approached by the mol fraction of the solute is the same whether solvation occurs or not. ${ }^{8}$

Owing to an "historical accident" the latter method of eliminating the complication of solvation has been the one followed by science, instead of the former and more logical one. The "historical accident"s in this instance was van't Hoff's brilliant discovery of the remarkably simple equation connecting osmotic pressure with temperature and concentration in very dilute solutions. Starting with this equation as a basis and using the principles of thermodynamics, he showed us how to construct a complete theory of dilute solutions.

As the field of dilute solutions became more and more developed, both from the experimental and theoretical side, investigators began to turn their attention to the subject of concentrated solutions. Investigation in this direction has usually taken the direction of attempts to extend the osmotic pressure equation by the introduction of quantities corresponding

7 This system has been consistently followed by all investigators who use systems of thermodynamics based upon Gibbs' thermodynamic potential. The reason that it has not been followed by others is because they have confined themselves to the region of dilute solutions, where it is possible to use one of the limiting forms approached by the mol fraction of the solute, as the concentration approaches zero.

${ }^{8}$ To illustrate, if we put $n^{\prime}$ mols of a solute in $n$ mols of a solvent and no solvation (or dissociation or association) occurs, then the mol fraction of the solute is $\frac{n^{\prime}}{\left(n^{\prime}+n\right)}$. If, however, on the average $x$ mols of solvent are combined with each mol of solute, the mol fraction of the (solvated) solute is $\frac{n^{\prime}}{\left[n^{\prime}+n-n^{\prime} x\right]}$ or $\frac{n^{\prime}}{\left[n^{\prime}(\mathrm{I}-x)+n\right]}$. As the solution becomes more and more dilute, both expressions approach $\frac{n^{\prime}}{n}$ as their limit. This is the familiar ratio which appears in our boiling point and freezing point equations. In the case of our osmotic pressure equation, instead of writing it $\pi=\left(\frac{n^{\prime}}{n}\right)\left(\frac{R T}{V}\right)$, where $V$ is the molecular volume of the solvent, it is customary to substitute $V_{s}=$ $n V$ and write $\pi V_{s}=n^{\prime} R T$. Here again, if the solution is sufficiently dilute, it is obviously immaterial whether we understand $V_{s}$ to mean the volume of the solution or the volume of pure solvent in which the $n^{\prime}$ mols of solute were dissolved in preparing the solution.

"The expression, "historical accident," is, of course, used in the philosophical sense. 
to the $a$ and $b$ of van der Waals' condition equation for gases, upon the basis of kinetic conceptions derived from an assumed analogy between osmotic and gas pressure. Other investigators have sought to attribute all of the deviation of concentrated solutions from the equations of dilute solutions, to solvation, and have even gone so far as to compute on this basis the approximate degree of hydration in some very concentrated aqueous solutions, for example. Still other attempts have been taken in the direction of an extension of our present equations by the addition of a series of terms containing a number of constants intended to express the influence of the solute molecules upon one another and upon the solvent.

Attempts to obtain a satisfactory theory of concentrated solutions in any of these directions give no promise of success. An attempt to "explain" why, as a solution becomes more and more concentrated, it deviates more and more from the equations of very dilute solutions is somewhat analogous to an attempt to explain why the sine of angle, which for sufficiently small angles is equal to the angle, deviates more and more as the angle grows larger. The reason is, of course, a purely mathematical one. Similarly in the case of solutions there is first of all a purely mathematical reason why concentrated solutions should deviate from the equations of the infinitely dilute solution. The equations of dilute solutions are the limiting forms assumed by more general equations, owing to the fact that certain terms become negligible as the concentration approaches zero. In other words we have in our dilute solution laws only a portion, the residue, so to speak, of a more general set of laws for solutions of all concentrations. Consequently before science can hope to make any progress in the region of concentrated solutions she must go back to the point where the simplifying assumption of a dilute solution was unconsciously introduced, and, in place of it, make the simplifying assumption of an "ideal solution" as we have defined it above. We come, therefore, to

\section{The Laws of the Ideal Solution.}

Owing to the simplicity of the thermodynamic treatment of solutions by what we may call the osmotic-cyclical-process method and the fact that it uses conceptions which are comparatively easy to grasp and processes which can be readily pictured in the mind, it has been the favorite system among physical chemists. The fact that the theories developed by the advocates of this method have been confined almost entirely to the domain of the dilute solution is not due to any inherent fault in the methoc. In addition to this method we have the systems of thermodynamics based upon the Gibbs thermodynamic potential and its related functions. These systems have been the favorite ones among physicists and those who by training and inclination were accustomed to the use of potential functions, and it is among the advocates of the thermodynamic potential that we find the first successful attempt to formulate a theory of solution which is free from the assumption that the solution must be dilute.

This theory has been developed in Holland by van der Waals and his associates, especially by van Laar. The first attempt was made in 1893 by Hondius Boldingh ${ }^{10}$ in an Amsterdam Dissertation which so far as I

${ }^{10}$ Boldingh, "De Afwijkingen van de Wetten voor Verdunde Oplossingen." Dissertation, Amsted dim, r893. 
have been able to learn has never been published elsewhere. In the following year van Laar ${ }^{11}$ published two papers in which he derived a set of "exact formulae for osmotic pressure, change in solubility, freezing point, boiling point, etc." His results were expressed in a series of equations in which the concentration of the solution appeared in a term, $\ln \left(\mathrm{I}-N^{\prime}\right)$, in which $N^{\prime}$ represents the mol fraction of the solute. The equations contained, in addition, an undetermined function of the molecular thermodynamic potentials of the constituents. In numerous subsequent publications van Laar has advocated with great warmth and zeal, the use of the thermodynamic potential method and the introduction of the concentration of the solution into the equations by means of the expression $\ln \left(\mathrm{I}-N^{\prime}\right)$, instead of assuming that the solution is dilute. Van Laar has in fact advocated a theory of solution which is entirely free from the assumption that the solution must be dilute. The foundations for this theory have existed in the literature for the last fifteen years. If it occurs to any one to wonder why the theory has not come into general use in the chemical world, he has only to glance through some of van Laar's papers, especially his earlier ones, and the reason will be more or less obvious.

It is my present purpose to free this theory from the language of the thermodynamic potential and to develop it in the so-called "osmotic language."12 Stated in this language, our problem is to determine the functional relation between some one of the colligative properties of the solution and its concentration in the case of an ideal solution. Theoretically we can start with any one of the colligative properties we choose, but since our present theory of dilute solutions is usually assumed to start with the osmotic-pressure-concentration relation, it will perhaps be more interesting to develop our theory of the ideal solution from the same standpoint.

Let us, therefore, turn to the equation which expresses the osmotic pressure $^{13}$ for a very dilute solution:

$$
\pi=\frac{n^{\prime} R T}{V_{s}}=\frac{n^{\prime} R T}{n V}
$$

In this equation, $n^{\prime}$ is the number of mols of solute in $n$ mols of solvent and $V$ is the molecular volume of the pure liquid solvent. Let us now make use of the method, introduced by Willard Gibbs, of expressing the composition of the solution by means of the equation $N^{\prime}+N=I$, where $N^{\prime}$ is the mol fraction of the solute and $N$ that of the solvent. The above equation can now be written:

$$
\pi=\frac{\frac{n^{\prime}}{n+n^{\prime}} R T}{\frac{n}{n+n^{\prime}} V}=\frac{N^{\prime} R T}{N V}
$$

11 van Laar, Z. physik. Chem., I5, 457 (1894).

12 The "language of the colligative properties" would be a better term. Too much importance is usually ascribed to osmotic pressure in our solution theory.

13 Throughout this paper, we shall understand by the term "osmotic pressure," the pressure difference $\pi$ as defined by the equation $\pi=P-P_{\mathbf{A}}$, where $P_{\mathbf{A}}$ is the pressure upon the pure liquid solvent $A$ when it is in equilibrium (through a membrane or medium permeable only to itself) with the solution under the constant pressure $P$. This has been discussed more fully in the previous paper (ThIs JourNal, 32, 478 (I910)). 
We have long recognized the fact that our osmotic pressure equation expresses strictly only the limit approached by the osmotic pressure as the concentration of the solution approaches zero. Let us therefore write the equation itself so that it will indicate this fact. This gives us

$$
\Delta \pi=\left(\begin{array}{c}
\Delta N^{\prime} \\
N
\end{array}\right)\left(\begin{array}{c}
R T \\
V
\end{array}\right)
$$

Seeing the equation in this form it is natural to suspect that the real relation might possibly be

or since by definition- $d N^{\prime}=d N$,

$$
d \pi=\left(\frac{d N^{\prime}}{N}\right)\left(\frac{R T}{V}\right)
$$

$$
d \pi=\left(\frac{-R T}{V}\right) d \ln x \text {. }
$$

Stated in words, this means that not only would the addition of $d N^{\prime}$ mols of solute to a pure solvent involve an increase (i.e., from o to $\Delta \pi$ ) of osmotic pressure which satisfies equation (104) but that it would also involve the same increase in osmotic pressure when added to a solution whose osmotic pressure is $\pi$. If such proves to be the case (and we shall see that in many cases, at least, it does), our Modern Theory of Solutions has remained a theory of infinitely dilute solutions, because we have failed to recognize the fact that we have been working with true differential equations, and that in order to obtain the "theory of concentrated solutions" which we have been seeking, the only thing we needed to do was to integrate our equations.

In the case of osmotic pressure, for example, if we integrate equation (IO4), we shall obtain an equation which contains no assumption whatever regarding the concentration. The solution may be infinitely dilute or infinitely concentrated or may have any concentration between these limits. In order to do this we have only to put $V=V_{0}(\mathrm{I}+\alpha \pi)$, where $V_{0}$ is the molecular volume of the pure solvent under the standard pressure $P$ and $\alpha$ is its coefficient of compressibility, and on integration we obtain the Boldingh-van Laar ${ }^{14}$ equation for osmotic pressure:

$$
\pi+T / 2 \alpha \pi^{2}=\left(\frac{-R T}{V_{0}}\right) \ln \left(\mathrm{I}-N^{\prime}\right)
$$

14 The history of this equation (105) is very interesting. The differential form as expressed by equation ( 104 ) was obtained by van der Waals as early as I89o ( $Z$. physik. Chem., 5, I63) but no attempt was made to integrate it, only the case of dilute solutions being discussed, for which case it assumes the form of equation (102) or (100). In 1893 Hondius Boldingh, a student of van der Waals, making use of the thermodynamic potential of Gibbs, derived equation (IO5) in the following form (Diss., Amsterdam, 1893, p 57):

$$
\pi V_{0}=-R T \ln \left(\mathrm{I}-N^{\prime}\right)+a N^{\prime}
$$

This differs from equation (IO5) as we have obtained it above, only in the fact that the compressibility of the liquid is neglected and a small correction term $a N^{\prime}$ is added, $a$ being a quantity which, according to the molecular theory of van der Waals, expresses the mutual influence of the components of the solution upon each other. For "ideal solutions" it is negligible.

Boldingh apparently made no attempt to apply his equation. The same equation was obtained the following year by van Laar (Loc, cit.) and in numerous publications 
Having come to the conclusion that the integral of equation (104) should represent the osmotic pressure for an "ideal solution," whatever its concentration, we naturally seek for experimental confirmation before adopting it finally. ${ }^{15}$ Owing to the great difficulty of making accurate and

since then, this investigator has given various derivations of this equation usually by methods involving the thermodynamic potential (cf., however, note 15 ). In I897 an osmotic pressure equation in its essential points practically identical with equation (I05) was derived by Willard Gibbs (Nature, 60, 46I (1897)) by a method of balanced columns. Finally G. N. Lewis, in a recent paper (ThIs Journal, 30, 675 (Igo8)), has obtained equation (105) by a derivation involving his "activity" function and based upon the assumption that the "activity" of the solvent is proportional to its mol fraction. Both van Laar (Proc. Acad. Sci., Amsterdam, 9, 55 (I906)) and Lewis (Loc. cit.) have discussed the relation of this equation to the van't Hoff equation and have made comparisons of the values of osmotic pressure given by it with those obtained by Morse and Frazer by direct measurement, in the case of aqueous solutions.

${ }^{13}$ In view of the fact that the van't Hoff equation for osmotic pressure is usually regarded as derivable from the kinetic theory by methods analogous to those used in the kinetic derivation of the perfect gas laws (that is, on the assumption that osmotic pressure is caused by the molecular bombardment of the solute molecules), it may not be without interest to include here a brief kinetic derivation of the differential form of equation (IO5). For this purpose I shall modify slightly the derivation given by van Laar (Sechs Vorträge, p. 20).

Consider two solutions of the same solute in the same solvent, both under the external pressure $P$ and separated from each other by a membrane permeable only to the molecules of the solvent. According to a theorem of Boltzmann, the number of solvent molecules which diffuse per second through a unit surface of the membrane in the two directions is given by the expressions:

(a) From the weaker solution to the stronger,

$$
n_{w}=\left(I-N_{w}^{\prime}\right) e^{\frac{i+P V_{w}}{R T}} \text {. }
$$

(b) From the stronger solution to the weaker,

$$
n_{s}=\left(\tau-N_{s}^{\prime}\right) e^{2+P V_{s}} \text {. }
$$

In these equations $e$ is the base of natural logarithms, $N^{\prime}{ }_{w}$ and $N^{\prime}{ }_{s}$ are the mol fractions of solute in the weaker and the stronger solutions respectively, $V$ is the volume of the solution, $R$ the gas constant, $T$ the absolute temperature and $\lambda$ a quantity which is a function of the temperature and which depends upon the units of measurement. By adjusting the pressure on the two solutions we can make the number of molecules of solvent which pass in the two directions equal; in other words the two solutions will be in equilibrium as respects the passage of the solvent from one to the other. Under these conditions the right-hand members of the above equations can be placed equal to each other, giving us the equation:

$$
\left(I-N^{\prime}{ }_{w}\right) e^{\lambda+P_{w} V_{w}} \frac{\lambda T}{R T}=\left(I-N^{\prime}\right) e \frac{\lambda+P_{s} V_{s}}{R T}
$$

Let us now impose the condition that the "weaker solution" shall be the pure solvent and that the "stronger solution" shall be an infinitely dilute solution in this solvent and shall be under an external pressure $P$. Under these conditions the above equation assumes the form

$$
e^{[\lambda+(P+d \pi) V]} \frac{\left(1-d N^{\prime}\right) e^{\frac{(\lambda+P V)}{R T}}}{R T} .
$$

Dividing through by $\frac{(\lambda+P V)}{R T}$ and using the logarithmic instead of the exponential 
reliable osmotic pressure measurements, it would be an unnecessary waste of time and effort to seek experimental confirmation in this direction, especially as the equation can be tested just as satisfactorily by means of its thermodynamic derivatives. Of these we will consider first, the vapor pressure derivative. The thermodynamic relation connecting osmotic pressure and vapor pressure is

nomenclature, we obtain equation ( 104$)$ :

$$
d \pi=\left(\frac{-R T}{V}\right) d \ln \left(I-N^{\prime \prime}\right)
$$

After giving a kinetic derivation for equation (105), van Laar follows it with what he terms a "rein thermodynamischer" proof. Such a proof is of course impossible, if by "purely thermodynamic" we are to understand that the equation can be shown to be a necessary consequence of the two laws of thermodynamics and nothing else. In his papers on the subject, van Laar does not distinguish carefully between purely thermodynamic relations and relations which involve additional assumptions. This makes it difficult for the reader, who has not had considerable experience in the use of the thermodynamic potential, to appreciate just what assumptions he is making and what grounds he has for making them. Van Laar also falls into the error of attributing the failure of the modern theory of solutions in the region of concentrated solutions, to an inherent weakness in the osmotic method which he condemns severely, at the same time advocating with great zeal the thermodynamic potential as the only quantity which is in a position to completely solve the problem (Sechs Vorträge, p. 19). This point of view is absolutely unjustified and is doubtless partially responsible for the fact that the many excellent and valuable features of this investigator's contributions to this problem have not received from the chemical world the consideration which they deserve. Whether we should adopt a system of thermodynamic chemistry based upon the entropy function (as worked out by Horstmann), or upon one of the thermodynamic potentials of Gibbs or Planck, or upon the "fugacity" and "activity" as defined by Lewis or upon the "osmotic pressure" and its related colligative properties, is largely a philosophical question in which the personal equation is an important factor. The "best" system from one point of view is not the "best" from another, and instead of adopting one of these systems and severely condemning the others, we should rather rejoice that the problems of our science are being attacked from these different points of view. All of these systems rest upon the common ground of the first and second laws of thermodynamics and any chemical problem which can be solved in terms of one of them can be solved in terms of all. I cannot therefore agree with van Laar, that the so-called "osmotic" system "läuft auf seinen letzen Beinen" and "nach wenige Jahre wird abgereist sein."

Van Laar also attacks the so-called "gas theory" of solutions, that is, the theory that what we call "osmotic pressure" is a real pressure which exists within an isolated solution due to a molecular bombardment by the solute molecules. On this question, I sympathize largely with van Iaar's point of view. His exposition of the difficulties in the way of such a theory is clear and convincing and I shall not, therefore, attempt any further discussion of the question at this time. In this connection, however, it is interesting to recall the views held by Willard Gibbs upon this point. In speaking (Loc, cit.) of the osmotic pressure in the case of a solution $A$, containing a solute, D, he says:

"But we must not suppose in any literal sense, that this difference of pressure represents the part of the pressure in $\mathrm{A}$ which is exerted by the $\mathrm{D}$-molecules, for that would make the total pressure calculable by the law of Boyle and Charles." 


$$
d \pi=\left(\frac{-v}{V}\right) d p
$$

Combining this with equation (IO4) so as to eliminate $\pi$ we obtain

$$
V d p=R T d \ln N \text {. }
$$

If the vapor can be regarded as a perfect gas we can put $v=\frac{R T}{p}$ and obtain

which on integration gives

$$
d \ln p=d \ln N
$$

$$
p=p_{0} N
$$

where $p_{0}$, the integration constant, is the vapor pressure of the pure solvent. Since the terms solvent and solute are quite arbitrary, we can state therefore in general that the partial vapor pressure of any constituent of an "ideal solution" is proportional to its mol fraction, if the vapor obeys Boyle's law. We have therefore in equation (II3) an excellent means of testing our fundamental osmotic equation.

The next question which confronts us is, where are we to look for solutions whose characteristics approach most closely those which we have assumed for our "ideal solution," or in other words where can we find solutions for which we have reason to believe that we know the mol fractions of the constituents in the solution? Our attention is naturally directed towards mixtures of the so-called "normal" liquids of which many examples are to be found among the hydrocarbons of the benzene series and their substitution products. These liquids possess the property of "mixing with each other in all proportions, the process of solution being accompanied by little if any heat effects or volume changes, such as would, in general, necessarily occur, if the process of solution were accompanied by chemical reactions such as solvation or changes in the degree of association or dissociation of any of the components. In general the physical properties of these solutions are additive with respect to the constituents. This behavior is; however, just what we should expect in the case of the "ideal solution" which we have assumed. We may therefore expect to find experimental confirmation of our osmotic pressure equation in the case of these solutions. Fortunately data are at hand in the vapor pressure measurements of Zawidski and others. These data show most conclusively that equation (II3) expresses the partial vapor pressure for both constituents throughout the total concentration range from zero to infinity for some dozen or fifteen different mixtures. ${ }^{16}$ Freez-

10 The mixtures which obey this vapor pressure law are as follows:

(1) $\mathrm{CO}_{2}-\mathrm{CH}_{3} \mathrm{Cl}$; (2) $\mathrm{C}_{6} \mathrm{H}_{14}-\mathrm{C}_{8} \mathrm{H}_{18}$; (3) $\mathrm{C}_{2} \mathrm{H}_{4} \mathrm{Cl}_{2}-\mathrm{C}_{6} \mathrm{H}_{6}$; (3) $\mathrm{C}_{2} \mathrm{H}_{4} \mathrm{Br}_{2}-\mathrm{C}_{3} \mathrm{H}_{6} \mathrm{Br}_{2}$; (2) $\mathrm{CH}_{3} \mathrm{OH}-\mathrm{C}_{2} \mathrm{H}_{5} \mathrm{OH}$; (2) $\mathrm{CH}_{3} \mathrm{COOC}_{2} \mathrm{H}_{5}-\mathrm{C}_{2} \mathrm{H}_{5} \mathrm{COOC}_{2} \mathrm{H}_{5}$; (2) $\mathrm{C}_{6} \mathrm{H}_{6}-\mathrm{C}_{6} \mathrm{H}_{5} \mathrm{CH}_{3}$; (4) $\mathrm{C}_{6} \mathrm{H}_{6}-\mathrm{C}_{6} \mathrm{H}_{5} \mathrm{Cl}$; (4) $\mathrm{C}_{6} \mathrm{H}_{6}-\mathrm{C}_{6} \mathrm{H}_{5} \mathrm{Br}$; (2) $\mathrm{C}_{6} \mathrm{H}_{8} \mathrm{CH}_{3}-\mathrm{C}_{6} \mathrm{H}_{5} \mathrm{C}_{2} \mathrm{H}_{5}$; (4) $\mathrm{C}_{6} \mathrm{H}_{5} \mathrm{CH}_{3}-\mathrm{C}_{6} \mathrm{H}_{5} \mathrm{Cl}$; (4) $\mathrm{C}_{6} \mathrm{H}_{5} \mathrm{CH}_{3}-\mathrm{C}_{6} \mathrm{H}_{5} \mathrm{Br}$; (2) $\mathrm{C}_{6} \mathrm{H}_{6} \mathrm{Cl}-\mathrm{C}_{6} \mathrm{H}_{5} \mathrm{Br}$.

References:

${ }^{1}$ Kuenen, Z. physik. Chem., I I, 38 (1893).

2 Young, J. Chem. Soc., 81, 768; 83, 68 (1903).

s von Zawidski, Z. physik. Chem., 35, 129 (1900).

- Linebarger, This JournaL, I7, 615, 690 (I895).

This experimental confirmation of the theory of the ideal or "perfect" solution was pointed out in a recent paper by G. N. Lewis (Loc. cit.) who has computed some 
ing point data furnish additional confirmation of the correctness of our fundamental equation. Van Laar finds, for example, that the "freezing point" curve for solutions of mercury in tin, throughout its entire range (from $t=232^{\circ} \mathrm{C}$, $N^{\prime}=0$ to $t=-19^{\circ}, N^{\prime}=0.9964$ ), is satisfactorily represented by an equation which rests on the same basis as our fundamental osmotic pressure equation.

This perfect experimental confrmation, combined with the light which is thrown upon the subject by the historical criticism, constitutes a most convincing array of evidence in favor of the adoption of the Theory of the Ideal Solution, as the best provisional General Theory of Solution. Before turning to a more detailed consideration of the equations of the Ideal Solution, let us state clearly the general characteristics of such a solution.

They are as follows:

I. The number of molecular species present is equal to the number of components.

II. The physical properties of the solution are connected with the physical properties of its components in the pure state by the equation

$$
x^{\prime}=x N+x^{\prime} N^{\prime}+x^{\prime \prime} N^{\prime \prime}+\ldots
$$

in which $X$ is the molecular property in question (e.g., molecular heat capacity, molecular volume, molecular refraction, molecular internal energy, etc. $), x\left(x^{\prime}, x^{\prime \prime}\right.$, etc. $)$ the molecular property of a constituent in the pure state and $N\left(N^{\prime}, N^{\prime \prime}\right.$, etc.) its mol fraction in the solution.

III. The third and most important characteristic is that which describes the thermodynamic relations. The manner of stating this characteristic depends upon what system of thermodynamics one chooses to make use of. I shall therefore state it in three different "languages."

(a) The Gibbs Thermodynamic Potential System.-According to van Laar the thermodynamic characteristics of the "ideal solution" are expressed by the equation

$$
\mu=\mu_{0}+R T \ln N
$$

in which $\mu$ is the molecular thermodynamic potential of a constituent in the solution, $\mu_{2}$ the molecular thermodynamic potential of the same constituent in the pure state and $N$ its mol fraction in the solution.

(b) The Fugacity-Activity System of Lewis.-Lewis uses a system of thermodynamic chemistry based upon two quantities, the "fugacity" $\psi$, and the "activity" $\xi$, whose relation to each other is expressed by the equation

$$
\varphi=\xi R T
$$

and which are connected with the thermodynamic potential by the equation

where $C$ is a function of the temperature only. According to Lewis the "perfect solution" is defined by the equation

or what amounts to the same thing,

$$
\xi=\xi_{0} N
$$

$$
\zeta=\psi_{0} N
$$

That van Laar's and Lewis' methods of 'describing the "ideal" or "perfect" solution are identical is made evident by writing equation (I I g) in the form

$$
R T \ln \psi=R T \ln \psi_{\circ}+R T \ln N,
$$

tables which exhibit in a very striking manner the excellent agreement of equation (I05) with the experimental data, even in the most concentrated solutions. 
and combining it with equation (II7) when we obtain at once equation (II5).

(c) The Colligative Property System or the So-called Osmotic System.According to this system, the relation between the colligative properties of the Ideal Solution and its composition is expressed by a set of equations which is composed of the equation

$$
d \pi=\left(\frac{-R T}{V}\right) d \ln N
$$

and its thermodynamic derivatives. ${ }^{17}$

Having established fundamental equations for the Ideal Solution, let us now derive a set of equations for such a solution similar to our present equations for dilute solutions, but free from any assumptions as to the concentration of the solution. In deriving such a set of equations we could start either with our osmotic pressure equation (IO4) or the vapor pressure derivative (II2). In either case we should obtain the same set of equations. There is not much reason for choosing one of these equations rather than the other as a starting point. Each possesses certain advantages for this purpose. In the following derivations, however, I shall start with the osmotic pressure equation (104) in each case. This procedure will allow of direct comparison with our corresponding derivations for dilute solutions and it moreover avoids the necessity of using the gaseous phase in the derivation of a relation which is independent of the properties of the vapor. The procedure for obtaining our set of equations is very simple. In each instance, it consists simply in combining equation (IO4) with the proper purely thermodynamic relation and then integrating the result. The purely thermodynamic relations have all been obtained by the author in the previous publication ${ }^{8}$ to which the reader is referred for their derivation. For convenience in reference these equations will be designated by the same numbers as in the preceding publication. All numbers below Ioo refer to the previous paper.

\section{Vapor Pressure.} is

We have already derived this equation for which the integrated form

$$
p=p_{0} N
$$

where $p$ is the partial vapor pressure of any molecular species from an ideal solution in which its mol fraction is $N$, and $p_{0}$ is its vapor pressure in the pure liquid state at the same temperature. For a single nonvolatile solute whose mol fraction is $N^{\prime}$ this can also be written in the form

$$
\frac{\Delta p}{p_{0}}=N^{\prime}=\frac{n^{\prime}}{\left(n+n^{\prime}\right)} \text {. }
$$

If in a mixture of say two liquids, polymerization of one or both constituents, or chemical combination between them occurs, we can obviously

${ }^{17}$ Regarding the general characteristics of the Ideal Solution as stated above, it should be noted that although in general the absence of heat effects or volume changes on mixing two liquids may be taken as evidence for the absence of accompanying chemical reactions, the reverse is not necessarily the case. Heat effects and volume changes may and doubtless do occur in the absence of any chemical reaction, although in such a case the heat effect will in the majority of cases be of quite a different order of magnitude from that which is caused by a chemical reaction. 
make use of equation (II3) to determine the exact nature and extent of these processes if we know the necessary partial vapor pressure data. Derivatives of equation (I I 3 ) for special cases of association and of chemical combination have been applied recently with considerable success by Ikeda $^{18}$ and by Dolezalek ${ }^{19}$ to the elucidation of the chemical condition of several pure liquids and of their mixtures. The accumulation of accurate and reliable vapor pressure, data is of the highest importance to a clearer and more complete knowledge of the nature of solutions. For reasons which $I$ have stated elsewhere, ${ }^{21}$ the vapor pressure equation is the relation best adapted to serve as a basis for the experimental attack on the problem of concentrated solutions.

\section{The Freezing Point Equation.}

If we combine the purely thermodynamic equation

$$
d \pi=\left(\frac{-L_{F}}{V}\right) \frac{d T_{F}}{T_{F}}
$$

with our fundamental equation

$$
d \pi=\left(\frac{-R T}{V}\right) d \ln N
$$

so as to eliminate $\pi$, we obtain the desired equation ${ }^{20}$

$$
d T_{F}=\left(\frac{R T_{F}{ }^{2}}{L_{F}}\right)\left(\frac{d N}{N}\right)=\left(\frac{-R T_{F}^{2}}{L_{F}}\right)\left(\frac{d N^{\prime}}{N}\right) .
$$

For very dilute solutions, we can of course substitute the freezing point lowering $\Delta t_{F}$ in place of $-d T_{F}$ and $\frac{n^{\prime}}{n}$ in place of $\frac{d N^{\prime}}{N}$ and obtain the familiar law of Raoult-van't Hoff for freezing point lowering in dilute solution:

$$
T_{0}-T_{F}=\Delta t_{F}=\left(\frac{R T_{0}{ }^{2}}{L_{F}}\right)\left(\frac{n^{\prime}}{n}\right)
$$

It is preferable, however, to integrate our differential equation and thus obtain a general equation for an ideal solution of any concentration. Before doing this we will substitute in equation (122)

$$
T_{o}-\Delta t_{F}=T_{F}, I-N_{*}^{\prime}=N \text { and }-d\left(\Delta t_{F}\right)=d T_{F}
$$

where $\Delta t_{F}$ is the freezing point lowering in centigrade degrees and $T_{0}$ is the freezing point of the pure solvent on the absolute scale. This gives us

$$
\frac{d N^{\prime}}{d\left(\Delta t_{F}\right)}=\frac{L_{F}\left(I-N^{\prime}\right)}{R\left(T_{O}-\Delta t_{F}\right)^{2}}
$$

In order to integrate, we must first express $L_{F}$ as a function of $\Delta t_{F}$ which is done by the following purely thermodynamic equation:

$$
L_{F}=L_{F o}-\Delta C_{P_{0}} \Delta t_{F}-1 /{ }_{2} \alpha\left(\Delta t_{F}\right)^{2}-1 /{ }_{3} \beta\left(\Delta t_{F}\right)^{3}-\ldots
$$

${ }^{18}$ Ikeda, J. Coll. Sci. Imp. Univ. Tokyo, 25, Art. ro (rgo8).

${ }_{18}$ Dolezalek, $Z$. physik. Chem., 64, 730 (I 908 ); see also Möller, Ibid., 69, 449 (1909).

${ }^{20}$ This equation was obtained by Boldingh (Loc, cit., p. $6 \mathbf{r}$ ) in the following form:

$$
\left(\frac{L_{F}}{R}\right)\left(\frac{d T_{F}}{T_{F^{2}}}\right)=\left[\frac{x}{\left(I-N^{\prime}\right)}+2 a N^{\prime}\right] d N^{\prime}
$$

where $a$ has the meaning explained in note (I4). Boldingh integrated his equation under the assumption that $L_{F}$ is independent of $T$. 
In this equation $L_{F_{0}}$ is the molecular heat of fusion of the pure solvent at its freezing point $T_{0}, \Delta C_{P_{0}}$ is the attendant decrease in the heat capacity of the system and $\alpha, \beta$, etc., are constants expressing the dependence of $\Delta C_{P}$ upon the temperature. Combining (85) with (125) we obtain finally (neglecting $\beta$ ):

$$
\frac{d N^{\prime}}{d\left(\Delta t_{F}\right)}-\frac{\left[L_{F_{0}}-\Delta C_{P_{0}}-1 /{ }_{2} \alpha\left(\Delta t_{F}\right)^{2}\right]\left(I-N^{\prime}\right)}{R\left(T_{0}-\Delta t_{F}\right)^{2}}
$$

This equation can now be integrated. A convenient way to do this is to integrate into a power series in the desired variable by applying McLaurin's theorem directly to the differential equation, carrying the series only so far as the accuracy of the experimental data warrant for the particular case under consideration. For example, in the case of water solutions if $\Delta t_{F}$ be known to $0.00 \mathrm{I}^{\circ}$, then for values of $\Delta t_{F}$ which do not exceed $7^{\circ}$, the terms containing $\alpha, \beta$, etc., are negligible and the application of McLaurin's theorem gives us the equation ${ }^{21}$

and

$$
N^{\prime}=\frac{L_{F o}}{R T_{0}^{2}}\left[\Delta t_{F}-1 / 2\left(\frac{L_{F_{0}}}{R T_{0}^{2}}+\frac{\Delta C_{P_{0}}}{L_{F_{0}}}-\frac{2}{T_{o}}\right)\left(\Delta t_{F}\right)^{2}\right]
$$

$$
\Delta t_{F}=\frac{R T_{0}^{2}}{L_{F_{0}}}\left[N^{\prime}+1 / 2\left(I+\frac{R T_{o}^{2} \Delta C_{P_{0}}}{L_{F_{0}}}-\frac{2 R T_{o}}{L_{F_{0}}}\right) N^{\prime 2}\right] \text {, }
$$

the remaining terms in the expansion not being significant.

If we desire to follow a freezing point curve through a considerable range of temperature, the general integral of equation (126) is more advantageous. The general integral is

$$
\begin{aligned}
R \ln \left(I-N^{\prime}\right)=\left(\Delta C_{P O}+\alpha T_{0}\right) \ln \frac{\left(T_{0}-\Delta t_{F}\right)}{T_{0}}- \\
\quad \frac{\left[L_{F O}-\left(\Delta C_{P O}+\alpha T_{0}\right) \Delta t_{F}+1 / 2 \alpha \Delta t_{F}{ }^{2}\right]}{\left(T_{0}-\Delta t_{F}\right)}+\frac{L_{F O}}{T_{0}} . \text { (129) }
\end{aligned}
$$

Equations of this general character have been derived by several observers ${ }^{22}$ and the corresponding theoretical curves have been compared with the experimental curves for a number of systems with good agreement. In these comparisons, however, the constants of the theoretical equation have been evaluated from the freezing point data themselves, so that the agreement loses a good deal of its significance.

\section{The Boiling Point Equation.}

By combining the purely thermodynamic equation

$$
d \pi=\left(\frac{L_{v}}{V}\right)\left(\frac{d T_{B}}{T_{B}}\right)
$$

${ }^{21}$ The application of equation (127) to the data for aqueous solutions and the interpretation of the results obtained have been discussed by the author in a previous paper (Technology Quarterly, 2I, 370 (Ig08)). This application was made on the assumption that the molecular weight of liquid water is I8. Although the results obtained on this assumption were fairly satisfactory up to a concentration of I molal, it is clear that a complete study of the behavior of aqueous solutions from the standpoint of the laws of the Ideal Solution must take into account the degree of association of the solvent. Further investigations along this line are now in progress in this laboratory.

${ }^{22}$ e. g., van Laar (Versl. K. Akad. van Wet., Amsterdam, 1903 and 1904; several papers) Roozeboom ("Die Heterogene Gleichgewicht," 2, 267 et. seq.), and Yamamota U. Coll. Sci. Imp. Univ. Tokyo, 25, Art. II (1908)). 
with equation (104) so as to eliminate $\pi$ we obtain the desired equation

$$
d T_{B}=\left(\frac{R T_{B}{ }^{2}}{L_{v}}\right)\left(\frac{d N^{\prime}}{N}\right)
$$

which for very dilute solutions takes the familiar form

$$
T_{B}-T_{B O}=\Delta t_{B}=\left(\frac{R T_{B 0^{2}}}{L_{v}}\right)\left(\frac{n^{\prime}}{n}\right) \text {. }
$$

In order to integrate the differential equation ( 130 ) we have only to express $L_{v}$ as a function of the temperature. The First Law of Thermodynamics gives us the rigorous equation

$$
\frac{d L_{v}}{d T}=c_{p}-C_{P}+\frac{L_{v}}{T}-\left(\begin{array}{c}
L_{v} \\
v
\end{array}\right)\left(\begin{array}{c}
\partial v \\
\partial T
\end{array}\right)_{p}
$$

by means of which we can calculate the temperature coefficient of $L_{v}$. If the vapor behaves as a perfect gas this equation becomes

$$
\frac{d L_{v}}{d T}=\Delta C_{P}
$$

and the methods of integration of equation ( 130 ) become perfectly analogous in every respect to those followed in the case of the freezing point equation in the preceding section. It is not necessary therefore to discuss them in detail. The final equations have the same form as the corresponding ones for the freezing point lowering.

\section{Chemical Equilibrium.}

Two examples will be sufficient to illustrate the method of derivation of the laws which regulate chemical equilibrium in the ideal solution. Let the equilibrium be expressed by the equation:

$$
a \mathrm{~A}+b \mathrm{~B}+\ldots \rightleftarrows m \mathrm{M}+n \mathrm{~N}+\ldots
$$

(a) The Effect of Concentration.-The purely thermodynamic equation for the effect of concentration upon chemical equilibrium in a liquid phase at constant temperature and pressure is

$$
-a V_{\mathbf{A}} d \pi_{\mathbf{A}}-b V_{\mathbf{B}} d \pi_{\mathrm{B}}-\ldots .+m V_{\mathbf{M}} d \pi_{\mathrm{M}}+n V_{\mathrm{N}} d \pi_{\mathrm{N}}+\ldots=0 .
$$

According to equation (IO4) the osmotic pressure for each substance taking part in the equilibrium is

$$
V \mathrm{X} d \pi_{\mathrm{X}}=-R T d \ln N_{\mathrm{X}}
$$

Combining these two equations we obtain the relation

$$
\frac{N_{\mathrm{A}}^{a} \cdot N_{\mathrm{B}}^{b} \cdots}{N_{\mathrm{N}}^{n} \cdot N_{\mathrm{M}}^{m} \cdots}=K_{N}
$$

where $K_{N}$ is a constant. This expression differs from the GuldbergWaage Law only in the substitution of the mol fraction $N$, in place of the volume concentration $C$. Equation (I36) was obtained by $\mathrm{Planck}^{23}$ as early as 1887 , and the reasons for adopting it in place of the GuldbergWaage form and for expressing the composition of solutions in terms of mol fractions instead of mols per liter were clearly stated by him at the same time.

(b) The Effect of Temperature.-The purely thermodynamic relation is

$$
-a V_{\mathrm{A}} d \pi_{\mathrm{A}}-b V_{\mathrm{B}} d \pi_{\mathrm{B}}-\ldots+m V_{\mathrm{M}} d \pi_{\mathrm{M}}+n V_{\mathrm{N}} d \pi_{\mathrm{N}}+\ldots=\frac{Q_{\pi} d T}{T} \cdot \text { (38a) }
$$

${ }^{23}$ Planck, Wied. Ann., 32, 489 (1887). 
Combining this with equations (136) and (135) and introducing the First Law of Thermodynamics we obtain the equation

$$
\frac{d \ln K_{N}}{d T}=\frac{U}{R T^{2}}
$$

which is identical with the van't Hoff Law, with the substitution of $\mathrm{K}_{N}$ in place of the Guldberg-Waage constant $K_{C}$. In general it may be stated that the laws for chemical equilibrium in an ideal solution may be obtained from our present dilute solution laws by substituting mol fractions in place of volume concentrations. This applies also to heterogeneous equilibrium. The Solubility Product Law, for example, for a saturated solution of the solute $\mathrm{BC}$ which dissociates into $\mathrm{B}$ and $\mathrm{C}$ becomes $^{24}$

$$
N_{\mathrm{B}} \cdot N_{\mathrm{C}}=\text { const. }=K_{S} \text {. }
$$

\section{Concluding Discussion.}

Lest any one from the perusal of the foregoing pages should gain the impression that the problem of a satisfactory general theory of solutions may be regarded as completely solved, it will be well to examine for a moment, in a general way, the characteristics of the Theory of the Ideal Solution with respect to its advantages and disadvantages when regarded as the basis for a general theory of solutions. When compared with the Theory of Dilute Solutions we must acknowledge that it constitutes a distinct and decided step forward. One requirement of a satisfactory general theory of solutions is that it shall represent the facts throughout the whole range of concentrations for some type of solution, at least. This requirement is fulfilled by the Theory of the Ideal Solution and we may feel considerable certainty that any deviation from the requirements of this theory, in a given case, is due to physical or chemical causes and capable of a physical or chemical explanation and is not simply the result of attempting to apply a set of incomplete laws which do not and could not be expected to hold for any kind of a concentrated solution no matter how simple its character.

The success of the Theory of the Ideal Solution as an instrument for throwing light upon the processes occurring in solutions has already been demonstrated in several instances. In the case of several solutions which apparently exhibit a behavior contrary to the requirements of the Theory, Dolezalek ${ }^{19}$ has shown that perfect agreement between theory and experiment exist if the assumption be made that a simple compound is formed between the two constituents or that one of them is partially asseciated. In the case of acetone and chloroform for example, the assumption of a single compound, $\mathrm{CHCl}_{3}\left(\mathrm{CH}_{3}\right)_{2} \mathrm{CO}$, and the introduction of the corresponding equilibrium constant into the equations sufficed to produce complete agreement between theory and experiment. It is true that the value of the constant was computed from the vapor pressure data themselves, but in a recent paper ${ }^{19}$ Möller has shown that the values of such constants may be obtained independently of the vapor pressure data of the solution under consideration and that they therefore possess

${ }^{24}$ This is obtained by combining equations (5I) and (I04). It does not involve the Mass Action Law [i.e., equation (136)], which is usually assumed as the basis for the derivation of the Solubility Product Law. This point, which was brought out in the previous paper, has been overlooked in all of the textbooks of physical chemistry, although it was explained clearly by Planck as early as 1887 (Loc. cit.). 
the physical significance ascribed to them and are not simply empirical constants of an interpolation formula.

In all the cases studied by Dolezalek he found that agreement between experiment and theory is produced if the assumption be made that what appears to be a deviation from the theory is simply due to the fact that the numbers assumed as the mol fractions of the two constituents in the solution are incorrect and that when the proper mol fractions are used, the apparent discrepancy disappears. If we were justified in assuming that all solutions are really ideal solutions and that what appear to be exceptions are merely due to our inadequate knowledge of the number and kind of the various molecular species present and their respective mol fractions, then the Theory of the Ideal Solution would constitute a general theory including all solutions and all concentrations and would enable us to ascertain just what occurs chemically, when the solution is formed out of its constituents. Unfortunately such is not the case, for it can be easily shown mathematically that if certain liquids form an ideal solution with one another they must be miscible in all proportions. The solutions in a system composed of two or more liquid phases in equilibrium with one another cannot therefore be governed to the laws of the Ideal Solution. Moreover, these exceptions are not merely apparent but are real and cannot be explained on the grounds of association, dissociation or chemical combination. The explanation must be looked for in a radical difference in the physical nature of the medium.

To illustrate by an extreme case, let us consider a system composed of a solution of benzene in mercury and a solution of mercury in benzene, both solutions in equilibrium with each other. The vapor pressure of benzene from the mercury layer is equal to its vapor pressure from the benzene layer and yet the mol fraction of benzene in the mercury layer is probably so small that we could not detect it by any analytical means, while in the benzene layer it is equal to $\mathrm{I}$, within the limits of our ability to measure it. The equality of the vapor pressure from the two layers can only be due, therefore, to the fact that the nature of the medium between the molecules of mercury is such that the benzene molecules can penetrate it only with the greatest difficulty. This effect of the physical nature of the medium is therefore one which must be taken account of in all applications of the Theory of the Ideal Solution. In order that the laws of the Ideal Solution shall apply, the nature of the medium or the field of force in which the molecules find themselves in the solution must not be very different from that of the pure liquid itself. Although this restricts somewhat the sphere of usefulness of the theory, there still remain a large number of cases where it should prove of the greatest value in the elucidation of the chemical nature of solutions. Even in cases where the theory cannot be extended over all concentrations because of a consequent radical change in the physical nature of the medium, we may still hope to obtain valuable results with its aid in solutions of moderate concentrations. At all events, I believe that the Theory of the Ideal Solution is the one which should be adopted as the basis for reference, classification and interpretation of the experimental data on solutions in place of our present Theory of the Infinitely Dilute Solution which is only a special, though very important, case of the former theory.

CEBAx, ILlinoIs, Feb. I, igio. 